\title{
Entscheidung als Häresie
}

\author{
Jaanus Sooväli \\ Institut für Philosophie und Semiotik, Universität Tartu
}

\begin{abstract}
Man könnte sagen, dass Aristoteles so etwas wie eine traditionelle und dominierende Auffassung der Entscheidung in der westlichen Philosophie gründet. Das Ziel dieses Aufsatzes ist es, diese Auffassung von Aristoteles zu entwerfen, einige ihrer wichtigsten Probleme anzusprechen, und sie damit zu kontrastieren, was ich Jacques Derridas häretische „Konzeptualisierung“ der Entscheidung nennen würde. Es wird gezeigt, dass diese häretische Thematisierung von Derrida nicht nur und einfach mit dem traditionellen aristotelischen Begriff bricht, sondern vielmehr auch zu Recht einige seiner wichtigsten Züge auf solcher Weise schärft, dass von diesem radikalisierten Standpunkt die Behandlung von Aristoteles als die Annullierung der Entscheidung zum Vorschein kommt. Es wird zudem impliziert, wie Derridas häretische Auffassung die Erneuerung der Ethik und Politik mit sich bringt.
\end{abstract}

Schlagwörter: Ethik, Entscheidung, Unentscheidbare, Freiheit, Verantwortung, Subjekt, Überlegung, Gesetz, Aporie

\section{Einführung}

Es scheint, dass es in der Philosophie des Abendlandes so etwas wie eine traditionelle Auffassung der Entscheidung gibt. Obgleich diese Tradition wie die abendländische Philosophie selbst - mehrere Linien aufweist und auch nicht homogen verläuft, lassen sich wiederkehrende Charakteristiken, Merkmale oder Züge auffinden, die man auch relativ klar beschreiben könnte. Viele von diesen Zügen können - wie ich im Folgenden zeigen werde bei Aristoteles gefunden werden. Es könnte sogar behauptet werden, dass Aristoteles als erster den sogenannten traditionellen Begriff der Entscheidung geprägt, ausgearbeitet und fortgeführt hat - er ist der Begründer und

Korrespondenzanschrift: Jaanus Sooväli, Institut für Philosophie und Semiotik, Universität Tartu, Ülikooli 18, Tartu 5009o, Estland. E-Mail: jaanus.soovali@ut.ee.

An abstract in English is available at the end of the article. 
Vertreter dessen, was man heute in der einen oder anderen Weise mit ,Entscheidung' in Verbindung bringt. Nun würde man vermuten, dass da, wo es eine Orthodoxie gibt, sich bei näherem Hinsehen auch so etwas wie eine Häresie auffinden lässt. Als häretisch werden Ansichten, religiöse Vorstellungen oder Meinungen bezeichnet, die unverträglich mit den anerkannten Wissens-, Religions- oder Meinungssystemen sind, diesen mehr oder minder gegenüberstehen oder auf andere Weise heterogen zu ihnen verlaufen. Man muss hier daran erinnern, dass die Bedingung der Möglichkeit von Häresie nur Orthodoxie selber sein kann - Häresie kann es nur in diesem Bruch mit und / oder Abbrechen von Orthodoxie geben, und solch ein Bruch beinhaltet immer auch Zugehörigkeit zur und Abhängigkeit von der Orthodoxie. $^{1}$

Es scheint, dass diese Auffassung von Häresie im Sinne von zugleich Bruch mit und Zugehörigkeit $z$ ur Tradition in den Werken Derridas im Hinblick darauf, was ,Entscheidung' ist, war oder sein kann, am deutlichsten hervortritt (aber auch woanders, zum Beispiel bei Kierkegaard). Aber nicht nur ist Derridas Thematisierung der Entscheidung oder Wahl häretisch die Entscheidung selber wäre nach ihm nur möglich als eine Art Häresie in Bezug auf die anerkannten Regeln und Normen, die die Orthodoxie leiten und strukturieren (vgl. Derrida 1995, 26). Häresie stammt gerade vom griechischen Wort hairesis ${ }^{2}$ Wahl' (vom Verb hairesthai ,zu wählen') ab. Es ist einigermaßen paradox, dass Aristoteles - wie schon gesagt einer der ersten, der den Begriff der Entscheidung thematisiert und zu einem Grundstein der Ethik macht - Entscheidung auf eine Weise versteht, die letztlich auf eine Annullierung und Aufhebung der Entscheidung als solche hinauszulaufen scheint, das heißt, die Entscheidung im Sinne von Zerreißen (decision), von einer Abweichung von Normen, Regeln, Überlegung etc. Man gewinnt den Eindruck, als ob er dieses Phänomen nur deswegen behandelt, um es aufzulösen und zu entschärfen (auf eine komplizierte und verfängliche Weise und sicherlich nicht direkt oder einfach), zumindest das Gefährliche und Grundlose darin. Ein erster, eher fadenscheiniger Hinweis darauf ist, dass

1 Dies schließt die Auflösung einer vermeintlich chronologischen Abfolge, in der die Orthodoxie traditionell den Vorrang behält, mit ein. Häresie könnte auch ,älter' als Orthodoxie sein, in dem Sinn, dass sich Glaubensmeinungen aus ursprünglichen Häresien entwickelten, sich durchsetzten und langsam zur Orthodoxie ausbildeten. So war das Christentum ursprünglich eine Häresie, und bis es zur Orthodoxie wurde, mussten in ihm selbst immer wieder Häresien bekämpft werden. Es hat aber, um sich auf der ganzen Welt auszubreiten, immer wieder auch häretisches Gedankengut und häretische Praktiken zugelassen oder gar aufgenommen. So scheint die Orthodoxie die Häresie genauso zur Voraussetzung zu haben wie die Häresie die Orthodoxie.

2 Das auch einen Akt des Nehmens bezeichnet, z. B. wenn Soldaten in eine Stadt stürmen, um sie zu erobern. 
Aristoteles nicht das Wort hairesis, sondern prohairesis (vom Verb prohairesthai) verwendet, wobei das Präfix „pro“ vor allem „früher" im Sinne eines Zugleich von Zeit und Vorzug impliziert, aber auch schlicht „vor“ bedeuten kann.

\section{Zur Etymologie von Entscheidung}

Offenkundig ist die Entscheidung, das Treffen einer Entscheidung mit der Wahl bzw. dem Wählen eng verknüpft, und auch die Denker, um die es in diesem Aufsatz gehen wird, haben diese beiden Begriffe nie wirklich voneinander getrennt und / oder die möglichen Unterschiede eigens thematisiert - „Entscheidung“ und „Wahl der Entscheidung“ bilden in gewisser Hinsicht ein Synonym. So kann auch im Griechischen krisis zugleich ,Entscheidung;, ,Wahl', ,Scheiden', ,Trennung', ,Streit', ,Urteil' bedeuten; das aristotelische Wort prohairesis meint ,Entscheidung', ,Wahl', ,Vorzugswahl'. Im Lateinischen steht für Entscheidung decisio (franz. décision, engl. decision), die vom Verb decido (de + caedo) ,abschneiden', abhacken' abgeleitet ist. Im Deutschen hingegen kann man vor einer Entscheidung stehen - in dem Sinn, dass es nur eine einzige gibt. Die Entscheidung besteht hier nicht darin, sich unter mehreren (Entscheidungs)alternativen zu entscheiden, sondern das Unausweichliche anzunehmen. Der heideggerische Eigentlichkeitsbegriff beruht im Wesentlichen darauf. Gleichwohl gibt es im Deutschen auch das ,Entscheidung treffen' als Wahl und es wird heute nahezu ausschließlich in diesem Sinn gebraucht.

Krisis, decisio, prohairesis und Entscheidung weisen alle auf das Scheiden, Zerreißen, Trennung und Aufschneiden hin. Was hat Entscheidung damit zu tun? Zum Ersten impliziert der tradierte Entscheidungsbegriff immer eine Unterbrechung, ein Aufschneiden, eine Freiheit als Vermögen, die einen neuen Anfang / eine erste Ursache erzeugt - etwas davon findet sich auch bei Aristoteles. Zum Zweiten könnte man das auf folgende Weise erklären: Am Anfang jeder Entscheidung muss schon eine bestimmte Scheidung oder das Abschneiden stattgefunden haben - nämlich eine Scheidung der bestimmten Phänomene, worüber man zuerst eine Entscheidung treffen wird. Beim wirklichen Entscheiden, beim Treffen der Entscheidung, wird aber noch ein Mal geschieden und abgeschnitten - das Entschiedene wird von dem abgeschnitten, wofür nicht entschieden wurde. Dieses Abschneiden oder Scheidung des Entschiedenen ist zugleich die Beseitigung der Scheidung zwischen mehreren Möglichkeiten, von denen eine ausgewählt wurde - sie ist, folglich, eine Ent-scheidung: ${ }^{3}$ das Wegschaffen der Scheidung

3 In Beiträgen der Philosophie fügt auch Heidegger einen Gedankenstrich in das Wort ein - er möchte damit aber „das Wesen des Seyns, ,, insgleichen die ontologische Differenz, 
selber. Demgemäß und paradoxerweise ist die notwendige Scheidung und das Abschneiden in jeder Entscheidung zugleich die Beseitigung und das Wegnehmen derselben Scheidung - für etwas wird entschieden und die Scheidung oder Trennung fällt dabei ab. Entscheiden bedeutet hier paradoxerweise so viel wie die Herstellung einer (umstrittenen) Einheit. Jedenfalls wirft das die Frage auf, ob dieses Scheiden und Abschneiden und zugleich die Beseitigung der Scheidung - die Ent-scheidung - streng von Gründen geleitet ist; ob es direkt aus dem Erwägen, Überdenken und Kalkulieren von Gründen resultiert und resultieren muss oder ob sich dieser Prozess irgendwie anders vollzieht. Von der Beantwortung dieser Frage hängt vieles ab. Möglicherweise setzt dieses Scheiden gerade eine Unterbrechung, einen neuen Anfang voraus. Oder anders ausgedrückt: Worin besteht das Verhältnis zwischen Entscheidung und dem Erwägen, Überdenken bzw. Kalkulieren von praktischen und theoretischen Gründen? Die verschiedenen möglichen Antworten auf diese Frage sind für das Auseinanderhalten der zwei Auffassungen der Entscheidung wesentlich, genauso wesentlich wie die Frage, wer eigentlich entscheidet - das heißt, gibt es (oder kann es geben und in welchem Sinne) tatsächlich einen rationalen oder sich seiner Irrationalität irgendwie bewussten Agent oder ein „Subjekt,,, der / das die Gründe überlegt und aufgrund dessen eine Entscheidung trifft? Dies sind einige Fragen, die wir uns durch Aristoteles und Derrida hindurch werden stellen müssen.

\section{Entscheidung als Ortho-doxie}

\subsection{Tugend als Basis der Entscheidung}

Im Grunde basiert die Entscheidung bei Aristoteles auf der Tugend (aretê). Im zweiten Buch der Nikomachischen Ethik $(N E)^{4}$ bestimmt Aristoteles die sittliche Tugend oder Tüchtigkeit als hexis proairetikê, wobei hexis eine feste Grundhaltung des Charakters bedeutet, die den wiederholten Handlungen entspringt, so dass, zum Beispiel, der Gerechte nur durch gerechtes Tun gerecht und der Besonnene nur durch besonnene Taten besonnen wird. Demgemäß ist hexis proairetikê mitunter (z. B. in der Oxford-Ausgabe, Aristotle 2002) als ,a disposition issuing in decisions" (eine Haltung, die in Entscheidungen resultiert) übersetzt worden (vgl. Broadie 2002, 43-45). Das Bedingende und das Bedingte nähern sich einander an. Nur: Wenn die Grundhaltung des Charakters in Entscheidungen resultiert, würde dies offensichtlich heißen, dass die Entscheidung eines Mannes oder einer Frau auf paradoxe Weise gerade darin bestehen würde, nicht so viel entscheiden zu müssen -

bezeichnen oder besser markieren (vgl. Heidegger 1989, 84-90; Cosmus 2001, 116-121).

${ }^{4}$ Im Folgenden wird Nikomachische Ethik als NE bezeichnet; Für alle Zitate aus diesem Werk habe ich die Reclam Ausgabe (Aristoteles 2006) verwendet. 
die erworbene Grundhaltung als Grundlage meiner sogenannten Entscheidungen bringt mir in meisten Situationen bei, was zu tun und was zu vermeiden ist, in einem Wort, sie bestimmt meine Taten und mein Verhalten. In der Tat, Aristoteles deckt explizit - zumindest in Bezug auf die unerwarteten und ungewöhnlichen Situationen - derartige Entscheidungen auf. ${ }^{5}$ Doch muss man sich nicht am Anfang dafür entscheiden, bestimmte Taten $\mathrm{zu}$ wiederholen, um diese hexis überhaupt und allererst zu erwerben und daraufhin (relativ sichere) Entscheidungen folgen zu lassen? Demnach wäre jene Grundhaltung oder Charaktereigenschaft nicht nur eine Grundlage der Entscheidungen und Taten, sondern auch das Resultat der prohairesis: man könnte gerecht oder mutig, wie gesagt, nur durch gerechtes und mutiges Tun werden (NE 1105a17-b12; vgl. Kuhn 1960, 134). Das wird in der Nikomachischen Ethik ziemlich deutlich gesagt - in dieser Hinsicht könnte hexis proairetikê als eine auf prohairesis beruhende Grundhaltung oder beruhender Habitus übersetzt werden. Andererseits dürfte man dabei nicht vergessen, dass Aristoteles eben der Erziehung eine ganz erhebliche Rolle bei der Aneignung dieser Grundhaltung (oder dem Erwerb des Habitus) zuschreibt - im Großen und Ganzen hängt es von der Erziehung ab, welche Handlungen man wiederholen und welche Grundhaltung daraus entstehen wird (NE 1103b4-b25). Aristoteles weist auch darauf hin, dass man am Anfang dem Vorbild, dem tüchtigen Menschen (phronimos) folgen müsse, um sich eine tugendhafte Grundhaltung anzueignen. Wenn aber die Aneignung der Grundhaltung nicht ganz frei und autonom verlaufen kann, muss man sie jedoch stets durch eigene Entscheidungen und Handlungen bestätigen und wieder bejahen (vgl. Derrida 1994, 36-37). Diese Bestätigung und Bejahung der Grundhaltung folgt einerseits aus der Grundhaltung selbst, andererseits muss sie durch Entscheidung und somit durch Erwägung / Überlegung hindurchgehen - zu dieser Beziehung zwischen Überlegung und Entscheidung kommen wir gleich. Zumindest lässt sich nicht leugnen, dass hexis und Entscheidung eng, vielleicht für das Entscheiden zu eng, miteinander verwoben sind.

Tugend kann freilich nicht nur und einfach als hexis proairetikê bestimmt werden (letztere ist Gattung der Tugend), weil dies die Grundhaltung der Schlechtigkeit einschließen würde. Deshalb fügt Aristoteles hinzu, dass Tugend als hexis proairetikê einen Ort einnimmt, der „die Mitte in Bezug auf uns ist, jener Mitte, die durch den richtigen Plan festgelegt ist, d. h. durch jenen, mit dessen Hilfe der Einsichtige [phronimos] (die Mitte) festlegen würde"(NE 1106b35-1107a2). Wichtig ist zu beobachten, dass die Definition der

5 Aristoteles schreibt: „Denn wenn etwas vorauszusehen ist, könnte man die Entscheidung auch berechnen oder durch nüchterne Überlegung herbeiführen, wenn dagegen etwas plötzlich hereinbricht, gibt die feste Grundhaltung den Ausschlag." (NE 1117a2O-24) 
sittlichen Tugend schon einen Hinweis auf die Verstandestugend beinhaltet - im sechsten Buch wird der „richtige Plan“ mit phronêsis, mit sittlicher Einsicht, mit der Fähigkeit zur richtigen Überlegung, gleichgestellt (NE 1144b26-27). Die sittliche Trefflichkeit oder Tugendhaftigkeit ist an sich nicht vollständig, sie braucht sittliche Einsicht (phronêsis), genauso wie letztere die Tugenden des Charakters benötigt (NE 1144b39-32; vgl. Ross 1985, 195). Die Richtigkeit einer Entscheidung wird durch beides gewährleistet und gesichert: „die Entscheidungen können nicht richtig sein ohne die sittliche Einsicht, aber auch nicht ohne die Trefflichkeit des Charakters, denn diese legt das Endziel fest, jene lässt uns die Mittel dazu ergreifen“(NE 1145a3-6). Sittliche Einsicht wird hierbei als zur Grundhaltung verfestigte Fähigkeit zum richtigen Überlegen beschrieben. So viel zum richtigen Erwägen / Überlegen. Was aber bedeutet die Überlegung im Allgemeinen (bouleusis) und wie verhält sie sich zur Entscheidung?

\subsection{Prohairesis und Überlegung}

Unmittelbar bevor er die Entscheidung im dritten Buch der Nikomachischen Ethik explizit und ausführlich behandelt, setzt sich Aristoteles verständlicherweise mit der Unterscheidung von Begriffen wie "freiwillig“ (hekôn) und „unfreiwillig“ (akôn) auseinander. Der traditionelle Begriff weist darauf hin, dass Entscheidung etwas freiwilliges sein muss. Die Problematik des freien Willens im tradierten Sinne tritt bei Aristoteles nur teilweise auf ${ }^{6}$ (vgl. Ross 1985, 201), für ihn ist vor allem wichtig, unter welchen Umständen man davon abzusehen bereit ist, jemanden für eine bestimmte Handlungsweise zu loben oder zu tadeln. Aristoteles fragt also nach den Gründen, die in der Gesellschaft als Entschuldigung akzeptiert werden könnten (Rapp 1995, 110). So könnte eine Handlung entschuldigt werden, die unfreiwillig geschah. Unfreiwillig nach Aristoteles ist nämlich das, was unter „Zwang (bia) oder aus Unwissenheit (agnoia) geschieht" (NE 1110a1).

Unter Zwang geschehende Handlungen sind solche, bei denen ein „bewegendes Prinzip von außen her eingreift, und zwar so, dass bei seinem Einwirken die handelnde oder die erleidende Person in keiner Weise mitwirkt: wenn z. B. jemand durch einen Sturmwind irgendwohin entführt wird oder durch Menschen, in deren Gewalt er sich befindet" (NE 1110a2-4). Aristoteles zufolge gibt es aber auch eine Art von gemischten Handlungen, wenn etwas ,aus Angst vor noch größerem Unheil oder für ein edles Ziel ausgeführt" (NE 1110a5) wird. Er bringt als Beispiel einen Tyrannen, der jemandem etwas Schlechtes zu tun befiehlt, „dessen Eltern und Kinder er in seiner

${ }^{6}$ Zum Beispiel haben Aristoteles zufolge auch die Tiere am Freiwilligen Anteil (NE 1111b510). 
Gewalt hat, und wenn diesen im Falle der Ausführung der Tat das Leben geschenkt, sonst verwirkt wäre ..." (NE 1110a5f). Diese Handlungen sind gemischt (gleichen aber mehr den freiwilligen), weil das bewegende Prinzip oder der Ursprung gerade im Augenblick der Handlung in der handelnden Person selber zu sein scheint; doch würde sie diese bestimmte Handlung natürlich nie wählen, wenn sie nicht dazu gezwungen wäre.

Wie angedeutet, kann auch Unwissenheit die Ursache von Unfreiwilligkeit sein, doch nicht Unwissenheit schlechthin - es gibt nämlich auch eine Art der Unwissenheit, die nicht die Ursache der Unfreiwilligkeit ist, z. B. die Unwissenheit aus Trunkenheit. An der letzteren trägt man selber Schuld und so können auch die Handlungen, die daraus folgen, nicht entschuldigt werden (weil nur die unfreiwilligen Handlungen entschuldigt werden können). Im Mittelpunkt soll hier die erste Form von Unwissenheit stehen, also jene, die Ursache von Unfreiwilligkeit sein kann und von Aristoteles näher bestimmt wird als Unwissenheit über konkrete Umstände und Gegenstände des Handelns. Diese Umstände sind Aristoteles zufolge (1) das ,Subjekt' der Handlung und das Bewusstsein von ihr (,... wer denn handelt und was er tut"), (2) der Gegenstand der Handlung, (3) die Mittel der Handlung, (4) der Zweck und (5) die Art und Weise der Handlung (z. B. ruhig oder heftig) (NE 1111a4f). Unwissenheit über diese Umstände kann die Unfreiwilligkeit verursachen und eine bestimmte Handlung entschuldigen. Nun habe ein Mensch immer (in mehr oder weniger großer Reichweite) Kenntnis über diese Umstände, es sei denn, er ist wahnsinnig. Im Folgenden fügt Aristoteles noch hinzu: „Selbstverständlich kann er auch nicht in Unwissenheit sein über die handelnde Person: wie könnte er sich selbst nicht kennen?" (NE 1111a7) Offensichtlich ist gerade dies ein für die tradierte Auffassung der Entscheidung zentraler Punkt. Ist es wirklich so selbstverständlich, dass der Entscheidende sich selbst kennt bzw. kennen muss? Könnte bzw. sollte der Handelnde oder Entscheidende nicht in einem gewissen Sinne in Unwissenheit darüber sein, wer eigentlich entscheidet und handelt? Und hat Entscheidung dann nicht auch etwas mit Wahnsinn zu tun? Wenigstens in Bezug auf Derridas Auffassung der Entscheidung müssen diese Fragen - wie wir noch sehen werden - mit aller möglichen Strenge gestellt werden.

Wenn als unfreiwillig dasjenige gilt, was unter Zwang oder / und aufgrund von Unwissenheit geschieht, ist umgekehrt bereits ein Kriterium für dasjenige, was freiwillig ist, gewonnen: „Dementsprechend darf als freiwillig das gelten, dessen bewegendes Prinzip in dem Handelnden selbst liegt, wobei er ein volles Wissen von den Einzelumständen der Handlung hat" (NE 1111a22-24). Entscheidung wird von Aristoteles hier als etwas Freiwilliges verstanden, doch er nimmt zugleich eine Einschränkung vor: nicht alles Freiwillige könne Gegenstand der Entscheidung sein - Entscheidung sei 
jener freiwillige Akt, dem Überlegung (bouleusis) voraufgegangen ist (NE 1112a15). Zieht man in Betracht, was bereits weiter oben in Bezug auf hexis proairetikê gesagt wurde, dann lässt sich hier eine bestimmte Spannung in der aristotelischen Auffassung der Entscheidung bemerken - eine Spannung bzw. gegenseitige Verschiebung zwischen Entscheidung verstanden als Ausdruck der festen Grundhaltung und Entscheidung verstanden als Abschluss der Überlegung. In jedem Fall wird die Entscheidung von der Überlegung bestimmt - Entscheidungen dürfen nicht einfach sein und schnell getroffen werden, man benötigt Zeit, um eine Handlung in ihren Konsequenzen und hinsichtlich ihrer Angemessenheit zu beurteilen (vgl. Derrida 1994, 33). Der Entscheidung muss jedoch die Überlegung nicht nur vorangehen, sondern sie muss direkt aus der Überlegung folgen; anscheinend ist sie sogar Ergebnis der Überlegung (vgl. Broadie 2002, 44). An dieser Stelle sollte man jedoch daran erinnern, dass Aristoteles zufolge nicht zu allem Überlegungen angestellt werden (und daher kann man sich auch nicht für alles entscheiden): man denkt nicht über die zeitlosen Dinge nach, über das, was mit naturgesetzlicher Notwendigkeit immer gleich bleibt, auch nicht über das, was immer anderes ist (z. B. das Wetter); man überlegt gleichfalls nicht das, wo es überhaupt keinen Zweifel gibt (z. B. wie die Schriftzeichen zu schreiben sind) und auch nicht über das Vergangene. „Wir überlegen uns das, was in unserer Macht steht und verwirklicht werden kann" (NE 1112a30-32). Überlegung weise dabei eine Ähnlichkeit mit analytischen und mathematischen Verfahren zur Lösung geometrischer Konstruktionsaufgaben auf (NE 1112b2of). Darüber hinaus richteten sich unsere Überlegungen nicht auf das Ziel, sondern auf die Wege zu diesem Ziel, und so entscheidet man auch nicht zwischen Zielen, sondern zwischen Wegen, die zu einem bestimmten Ziel führen (NE 1112b12f; 1113b2-5). Dieser letzte Punkt ist z. T. stark kritisiert worden (denn was wird in diesem Fall z. B. aus der Verantwortung?). Ich würde an dieser Stelle nur darauf hinweisen, dass es letzten Endes auf die Beantwortung der Frage ankommt, inwiefern der Erwerb der Grundhaltung und der Tugenden in unserer Macht steht, zumal Aristoteles im sechsten Buch unstrittig behauptet, dass das Ziel von der Grundhaltung gegeben oder bestimmt sei, und wenn nun die Entscheidungen eine Rolle bei der Erwerbung der Grundhaltung spielten, stünden natürlich auch die Ziele in einem bestimmten Sinne in unserer Macht (vgl. Rapp 1995, 127 ff.). Doch wegen der Wichtigkeit und der Bedeutung von Erziehung und Vorbildern könnte diese Rolle der Entscheidung scheinbar eher unwesentlich sein.

Was überlegt und entschieden wird ist nach Aristoteles eigentlich dasselbe, aber im Unterschied zur Überlegung ist die Entscheidung scharf abgegrenzt und festgelegt, sie enthält schon eine Festlegung (NE 1113azf) - 
Entscheidung ist nämlich dasjenige, was aufgrund von Überlegung vorgezogen wird. Und gerade in Bezug darauf tritt eine bestimmte Komplikation ein - man könnte nämlich einwenden, wie die Entscheidung als Festlegung überhaupt zustande kommt. Ist das Überlegen strukturell nicht etwas Unendliches? Aristoteles selber schreibt: „Falls ein Mensch unaufhörlich hin und her überlegen wollte, müsste er ins Endlose geraten" (NE 1113a2). Aristoteles könnte an dieser Stelle, wie Christof Rapp es versteht, einen Begründungsregress meinen - z. B. fragt sich der Entscheidende oder Handelnde vor der Tat „Warum die Handlungsweise A? Weil B. Warum B? Weil C, usw“ (Rapp 1995, 123 f.). Nur wenig weiter unten fügt Aristoteles hinzu: „Denn jeder hört auf zu suchen, wie er handeln soll, sobald er das bewegende Prinzip auf sich selbst zurückgeführt hat, und zwar auf den Teil seines Selbst, der die Führung hat: dieser Teil ist es, der die Entscheidung fällt" (NE 1113a5-7). Was heißt es, das bewegende Prinzip auf sich selbst zurückzuführen? Wie ließe sich das anstellen, wenn die Überlegung strukturell unendlich ist? Nach der Interpretation von Christof Rapp meint Aristoteles zunächst, dass der Handelnde zu einem Handlungsursprung werden muss, um den angesprochenen Begründungsregress zu vermeiden und die Entscheidung treffen zu können. Wie aber wird man zum Ursprung seiner Handlung? Setzt das gerade nicht den Bruch mit der Überlegung voraus? Man könnte den Eindruck haben, dass Aristoteles diese Schwierigkeit auf eine bestimmte Weise gesehen hat - zum Abschluss der Thematik und als Zusammenfassung beschreibt er die Entscheidung als „ein überlegtes Streben (orexis) nach dem, was in unserer Macht steht, denn nachdem wir etwas hin und her überlegt haben, treffen wir eine Wahl und streben dann entsprechend der (vorhergegangenen) Überlegung" (NE 1113a10-12). Folgt man Aristoteles, dann weist Entscheidung einen doppelten Ursprung auf: einerseits in orexis, das heißt im Streben oder in irgendeiner motivierenden bzw. treibenden Kraft, „im Dunkel des Nicht-Vernünftigen“ (Kuhn 1960, 126), und andererseits in Überlegung, das heißt in logos, im Vernünftigen, in der Vernunft selber. ${ }^{7}$ Nun das Unvernünftige, diese treibende Kraft richtet sich, wie er im Weiteren schreibt, an der Überlegung aus, sie wird geleitet und geführt von der Überlegung, von logos (vgl. Rawls 1993, 212. Das Streben strebt der Überlegung entsprechend, oder, wie Helmut Kuhn es ausdrückt: „Was der Logos bejaht, das erstrebt der Trieb (orexis) ..." (Kuhn 1960, 126). Der Eindruck verstärkt sich, dass Aristoteles die Entscheidung auf die Überlegung reduziert, obwohl er in einem bestimmten Sinne erkannt hat, dass es einen Bruch zwischen beidem geben muss. Streng genommen würde das

7 Vgl. mit Worten des scholastischen Kommentators Sylvester Maurus (1668:62): „... electio est appetitus consequens consultationem; et quia consultatio est actus rationis, ideo electio est appetitus cum ratione." 
Problem des Begründungsregresses immer noch bleiben - wie kann man sich entscheiden, wenn die Überlegung unendlich und das Streben darauf reduziert ist? Doch wahrscheinlich intendiert Aristoteles einfach Folgendes: Nachdem durch Überlegung allgemein bestimmt wurde, was getan werden soll, um irgendein Ziel zu erreichen, nimmt man wahr, was hier und jetzt, in diesem Augenblick und in dieser Situation, getan werden kann, so dass die Wahrnehmung der gegebenen Situation oder Umstände die Überlegung in einem bestimmten Sinne - und zusammen mit orexis, dem Trieb - zum Abschluss bringen würde (NE 1113a2-3; vgl. Ross 1985, 199). Da es jedoch mehrere Dinge geben kann, was man hier und jetzt durchführen könnte, müsste die Überlegung wieder ansetzen. Jedenfalls kann man Folgendes sagen: die Überlegung reicht nach Aristoteles nicht aus, um eine Entscheidung treffen zu können. Dazu bedarf es des Triebes, aber auch der Trieb wird in der Folge auf die Überlegung reduziert. Aristoteles hat damit einerseits einen Ursprung des Handelns offen gelegt, andererseits bleibt die Frage, wie dieser Ursprung hinsichtlich der Handlung, die er hervorbringt, aktiv werden soll.

Was soll man in diesem Fall davon halten, dass Aristoteles, obwohl nicht direkt im dritten Buch, das wir gerade behandelt haben, der Überlegung (genauso wie der richtigen Überlegung, phronêsis) eine syllogistische Struktur zuschreiben will (vgl. NE 1142a2o-22, 1142b21-26, 1144a31-33, 1147a25-31; vgl. Kuhn 1960; Ross 1985; Schmidt 2006, 333)? Gemeint ist kein theoretischer, sondern ein praktischer Syllogismus. Unter Syllogismus im Allgemeinen meint Aristoteles „eine Rede, in der, wenn etwas gesetzt wird, etwas von dem Gesetzten Verschiedenes notwendig dadurch folgt, dass dieses ist" (Aristoteles 1995, 2: 24b 18-20). Das mit Notwendigkeit sich Ergebende ${ }^{8}$ scheint beim praktischen Syllogismus nicht Satz, sondern eine Handlung zu sein (vgl. NE 1147a29-30). Praktischer Syllogismus hat einen allgemeinen Obersatz - z. B. vom Süßen sollte man kosten - und einen partikulären Untersatz - dies hier ist süß (NE 1147a3o). Man sollte hierzu anmerken, dass das zu erreichende Ziel und die Wege dazu kontingent sind, d. h. von bestimmten Situationen abhängen. Wenn nun aus beiden Prämissen eine einzige geworden ist, so würde man Aristoteles zufolge mit Notwendigkeit (anankê) die Folgerung in die Tat umsetzen, d. h. das Süße kosten (NE 1147a30-31). Obwohl Aristoteles einschränkend hinzufügt, „wer dazu in der Lage und nicht gehindert ist" (1147a3o), bleibt diese Behauptung, wie Richard Robinson $(1995,191)$ bemerkt, ziemlich steil und sokratisch. Werden die beiden Prämissen syllogistisch aufeinander bezogen, dann würde die Tat mit Notwendigkeit folgen. Was würde aber in diesem Fall aus der Entschei-

${ }^{8}$ Nach Helmut Kuhn wird der praktische Syllogismus von anderen Arten von Syllogismen gerade dadurch unterschieden, dass er nicht mit Notwendigkeit folgt (Kuhn 1960, 139). Doch im siebten Buch verwendet Aristoteles gerade das Wort anankê, , mit Notwendigkeit. 
dung werden? Ist sie nicht vollständig aufgelöst und aufgehoben? Zwar fügt Aristoteles, wenn er in der Nikomachischen Ethik vom praktischen Syllogismus spricht, jenes „mit Notwendigkeit“ nicht immer hinzu. Und er erkennt auch explizit eine (zweite) Art von phronêsis an, die nicht unbedingt eine solche syllogistische Struktur aufweisen muss - ältere Männer z. B., die schon eine bestimmte Erfahrung im Leben haben, wissen, was gemacht werden soll, sie haben eine gute Kenntnis von Einzelheiten und praktischen Situationen, obwohl sie die allgemeinen Prinzipien, den Obersatz des praktischen Syllogismus, möglicherweise nicht formulieren können (Ross 1985, 219). Daher muss man auf die Aussprüche der Älteren, „auch wenn sie ohne Beweis vorgetragen werden, genauso hören wie auf Beweise“ (NE 1143b10-14).

Wie weit oder eng man diese „Notwendigkeit“ im praktischen Syllogismus auch interpretiert, für sich genommen scheint er eine auf die Spitze getriebene und darin extreme Reduktion der Entscheidung auf die Überlegung zu sein - eine Reduktion, die schon im dritten Buch einigermaßen vorbereitet wird, worauf wir mehrmals hingewiesen haben. Selbst dann, wenn man diese "Notwendigkeit" nicht ganz wörtlich nehmen dürfte und wenn sie nicht völlig im Einklang mit dem, Geist' der aristotelischen Ethik stünde, weist der Text immer noch eine Neigung zu einer solchen radikalen Reduktion auf.

So halten wir einige Dinge fest: (1) die Entscheidung ist als ein Ausdruck der festen Grundhaltung beschrieben und (2) im Ganzen als Abschluss der Überlegung verstanden, auf Überlegung reduziert worden. Oder anderes ausgedrückt: einerseits ist die Entscheidung von der Grundhaltung, andererseits von der Überlegung bestimmt worden. Zwar scheint es eine Spannung zwischen diesen zwei Positionen zu geben, doch die Ähnlichkeit besteht vor allem darin, dass beide Einsichten dazu neigen, die Entscheidung $\mathrm{zu}$ verringern und in ihrer ursprünglichen Gewalt lindern. Wenn nun (3) der Überlegung darüber hinaus eine syllogistische Struktur zugeschrieben wird, so dass aus bestimmten Prämissen eine Tat mit Notwendigkeit folgt, ist die Entscheidung schlichtweg beseitigt. Außerdem (und demzufolge) ist (4) die Person, die sich für etwas entscheidet, nach Aristoteles nicht in Unwissenheit über sich selber, sie kennt sich selber und weiß, dass sie entscheidet; insgleichen kennt sie die Bedingungen der Entscheidung (d. h. was sie macht, zu welchem Zweck usw.) Und (5) ist die Richtigkeit der Entscheidung sowohl von der ethischen Tugend als auch von der dianoetischen Tugend, von phronêsis (,sittliche Einsicht' ${ }^{\top}$, gewährleistet. ${ }^{9}$

9 In Hinsicht auf die „erste Freundschaft“ hat Derrida an einer Stelle direkt, doch eher kurz und beiläufig, Bezug auf die aristotelische Konzeption der Entscheidung genommen. Die „erste Freundschaft“ beruht bei Aristoteles auf bebaios, was ,stabil', aber auch ,zuverlässig' heißt (vgl. Aristotle 1935, 380: 1237b 10-15) Bebaios als etwas Stabiles läuft auf Stabilisierung 


\section{Entscheidung als Ketzerei}

Derridas ,Konzeption' der Entscheidung bricht nun mehr oder minder radikal mit allen aufgelisteten aristotelischen Charakteristiken. Es sollte vielleicht gleich erwähnt werden, dass Derridas Auffassung mit vielen anderen Fragen und Themen - wie Verantwortung, Ereignis, Gabe, Gastfreundschaft, Vergebung usw. - eng verflochten ist und am Ende auf die Erneuerung der Politik (der Demokratie), der Ethik und des Rechtssystems hinausläuft: Die Entscheidung (wie auch Verantwortung und Gabe), auf neue und andere Weise verstanden, würde nach Derrida die Zukunft der Politik und Ethik offenhalten - Zukunft freilich nicht als eine Art Reproduktion der Gegenwart, future present, sondern gerade als Zu-kunft (à venir) verstanden. Nicht alle diese Beziehungen können im Folgenden zufriedenstellend hergestellt werden; es sollte aber deutlich werden, wie viel hier auf dem Spiel steht.

Im Hinblick darauf, dass Aristoteles die Entscheidung, den Passus der Entscheidung, von allen möglichen Seiten (ab)sichern wollte, könnte man an dieser Stelle an die Etymologie des Wortes "sicher" (secure) erinnern es stammt vom lateinischen sē-cūrus (sine cura), ,ohne Sorge, sorglos, rücksichtslos, ohne Angst und sogar ohne Verantwortung. ${ }^{10}$ Durch seine radikale Befragung zentraler Argumentationslinien bringt Derrida eben eine erhebliche Unsicherheit - und somit Angst, Verantwortung, Krisis - in die Entscheidung hinein. Entscheidung ist für ihn dasjenige, was mit dem Maschinellen, mit der Sicherheit der Überlegungen, Kalkulationen und Kausalitäten brechen sollte.

Im Vorwort von Politiques de l'amitié wirft Derrida die folgende Frage auf:

Nous nous demanderons alors ce quest une décision et qui décide. Et si une décision est, comme on nous le dit, active, libre, consciente et volontaire, souveraine. Que se-passerait-il si nous gardions ce mot et ce concept mais en changions ces dèrniers déterminations? (Derrida 1994, 15 f.)

Schon diese Frage weist auf die befremdliche Tatsache hin, dass Entscheidung bei Derrida nicht einfach als Ergebnis der Überlegungen eines

hinaus - man muss überlegen, erwägen, prüfen und kalkulieren, wer der beste Freund ist. Über bebaios schreibt nun Derrida: „Mais il marque aussi, ou plutôt il dissimule en le marquant, le passage entre deux ordres absolument hétérogènes, le passage de la certitude assurée, de la fiabilité calculable à la fiabilité du serment et de l'acte de foi. Ce dernier appartient, il doit appartenir à l'incalculable de la décision.... Cette structure est à la fois reconnue et méconnue par Aristote“ (Derrida 1994, 33 f.).

${ }^{10}$ Für die positive Rolle der Angst in Politik und politischen Entscheidungen, vgl. Sokoloff 2005. 
(selbst)bewussten, aktiven und sich selbst kennenden ,Subjekts' verstanden werden kann. Vielmehr fängt seine Auffassung gerade mit der kritischen Befragung dieser ,Subjektivität ${ }^{`}$ an. Mit Subjekt ist hier nichts anderes gemeint als eine bestimmte, mehr oder minder sichere ,Instanz', die den Entscheidungen untergelegen (sub-jectus) ist. Diese, Subjektivität' beinhaltet die Fähigkeit des Überlegens, die feste Grundhaltung (gewisse Identität), Bewusstheit und offensichtlich das Anderes-Handeln-Können, d. h. eine bestimmte Freiheit und Aktivität. Geht man der derridaischen Befragung des, Subjektes' nach, so zeigt sich zugleich, dass der Stellenwert der Überlegung beim Entscheiden immer schwieriger zu fassen ist und zum Schluss hin paradox wird - die Entscheidung darf Derrida zufolge gerade nicht auf die Überlegung und Gründe reduziert werden. Ich wende mich zuerst dem letztgenannten Punkt bzw. Charakteristikum zu.

\subsection{Heimsuchung des Unentscheidbaren}

Eingangs wurde darauf hingewiesen, dass als einer der Vertreter der - wenn man so sagen kann - häretischer Tradition der Entscheidung auch Kierkegaard genannt werden kann. Im dritten Kapitel von Donner la mort (1993) thematisiert Derrida die ,Begriffe' Verantwortlichkeit und Entscheidung explizit anhand von Furcht und Zittern. In diesem Buch versucht Kierkegaard eine Interpretation der biblischen Erzählung von Abraham, in der er die Frage beantworten will: Was soll das eigentlich bedeuten, dass Abraham im Christentum einerseits als Vater des Glaubens verehrt wird, andererseits, unter ethischem Gesichtspunkt, als (potentieller) Mörder bezeichnet werden könnte?

Bekanntermaßen befahl Gott Abraham, seinen Sohn Isaak, den sich er und seine Frau Sarah sehnlichst gewünscht und den sie erst in einem hohen Alter Sarahs wie durch ein Wunder oder als Geschenk erhalten hatten, auf dem Berg Moriah zum Opfer zu bringen. Abraham schweigt, spricht mit niemandem darüber, doch scheint er an Gott nicht zu zweifeln und nimmt am nächsten Morgen seinen Sohn und zwei Diener, um sich auf den Weg zum Berg Moriah zu machen. Isaak fragt seinen Vater mehrmals, wo das Lamm sei, das geopfert werden solle, worauf Abraham kurzerhand antwortet, dass Gott das Lamm besorgen werde. Im letzten Augenblick, als Abraham auf dem Berg Moriah bereits das Messer erhoben hat, um Isaak, seinen geliebten und lang erwarteten Sohn, zum Opfer zu bringen, erscheint ein Engel und hindert Abraham daran, ihn zu töten.

Diese Geschichte enthält Kierkegaard zufolge eine teleologische Suspension des Ethischen, d. h. das Ethische, das ein telos für menschliches Handeln bilden soll, wird für diesen einen Moment von Abraham suspendiert. Das Ethische ist das Allgemeine - das allgemeine Gut und Böse sowie die 
darauf beruhenden Regeln und Vorschriften, die der Einzelne in der Gesellschaft einhalten muss (vgl. Kierkegaard 1992, 49). Der Einzelne muss sich in seinen Entscheidungen an dem allgemein Guten und Bösen und den damit verbundenen Vorschriften orientieren. Im ethischen Allgemeinen muss der Einzelne seine Handlungen und Entscheidungen vor den anderen rechtfertigen, er muss das Geschehen rekapitulieren und Gründe für seine Handlungsweise angeben, er muss sich erklären, kurzum - er ist zum Sprechen aufgefordert. Im ethischen Sinne sollte Abraham seinen Sohn mehr lieben als sich selbst, er sollte diese Liebe noch über seinen Glauben stellen. Abraham aber scheint seinen Entschluss in keinem Augenblick ethisch in Zweifel zu ziehen und schweigt über die ganze Angelegenheit. Abraham unternimmt überhaupt nicht den Versuch, seine Entscheidung, seinen Sohn zum Opfer zu bringen, zu rechtfertigen, z. B. vor Sarah oder Isaak. Er nimmt die Gelegenheit, sein Vorhaben durch den Glauben zu rechtfertigen, nicht wahr. Indem Abraham nicht spricht, sich also nicht im allgemeinen Medium ausdrückt, nimmt er die absolute, und d. h. vereinzelnde Verantwortung gegenüber Gott auf sich. ${ }^{11}$ Wer würde ihn aber auch verstehen, und was hätte er eigentlich zu sagen? Er selber weiß fast nichts. Er weiß nur, dass Gott, das vollkommen Fremde, ihm befohlen hat, seinen Sohn zu töten. Er weiß nicht, warum (Gott gibt seine Gründe nicht an), und er kann sich nicht einmal sicher sein, ob tatsächlich Gott gesprochen hat (es könnte auch ein Dämon gewesen sein oder nur eine Halluzination). Er kann sich auch nicht darüber sicher sein, den ganz Anderen (Gott) ${ }^{12}$ richtig verstanden zu haben, er ist durch eine Unendlichkeit von ihm getrennt. Solange Gott ihm nicht sagt, dass er richtig verstanden habe, solange bleibt er in Ungewissheit darüber. Abraham weiß fast nichts - und trotzdem und ohne Zweifel hat er sich entschieden, seinen Sohn zu opfern. Abraham trifft eine Entscheidung, die sich anscheinend an keinen allgemeinen Normen und Regeln, an keinem allgemeinen Gut und Böse orientiert und somit auch nicht gerechtfertigt werden kann. Aus aristotelischer Sicht handelt es sich um eine augenblickliche und ungerechtfertigte Entscheidung aus Unwissenheit und ohne erkennbare Gründe.

Diese Art von Unwissenheit, durch die sich die Entscheidung Abrahams charakterisieren lässt, ist Derrida zufolge wesentlich für jede Entscheidung. ${ }^{13}$

${ }^{11}$ In Bezug auf wichtige Entscheidungen schreibt Aristoteles hingegen: „Zu Entscheidungen von großer Tragweite ziehen wir Ratgeber bei, weil wir uns nicht die Kraft zutrauen, allein zu einer Lösung zu kommen“ (NE 1112b1o-b12).

${ }^{12}$ Gott, wie man sieht, ist hier freilich nicht als Tyrann zu verstehen, der befiehlt und bestraft; Außerdem ist Gott in dieser Geschichte für Derrida nur ein Beispiel, der „ganz Andere“ ist jede Person.

${ }^{13}$ Ich möchte hier einem möglichen Einwand entgegentreten: Es könnte der Eindruck entstehen, dass es bei Kierkegaard und Derrida um die wichtigen, „existenziellen“ Entschei- 
In einer Sprache ausgedrückt, die auch Derrida selber mitunter verwendet, ist Unwissenheit die Bedingung der Möglichkeit des Sich-Entscheidens. In Donner la mort schreibt er:

His [Abraham's] non-knowledge doesn't in any way suspend his own decision, which re-mains resolute.... He accepts his responsibility by heading off towards the absolute request of the other, beyond knowledge. He decides, but his absolute decision is neither guided nor controlled by knowledge. Such in fact is the paradoxical condition of every decision: it cannot be deduced from a form of knowledge of which it would simply be the effect, conclusion, or explication. (Derrida 1995,77$)$

Hier bemerkt man schon das Häretische der Entscheidung, deren Bedingung es nach Derrida ist, nicht aus (nachvollziehbaren) Gründen, aus dem Wissen, aus kalkulatorischen Überlegungen, ethischen Vorschriften etc. abgeleitet werden kann. Warum nicht? Wenn die Entscheidung auf mehr oder weniger direkte Weise aus den Gründen, die man durch Überlegungen und / oder Erwägungen eruiert, folgen würde, wäre sie einfach keine Entscheidung mehr, und insbesondere keine verantwortliche Entscheidung, das will sagen: gäbe es ein sicheres Wissen und Gründe, dann befände man sich in einer Lage, in der es nichts zu entscheiden gibt und man sich daher auch nicht entscheiden muss. Eine solche „Entscheidung" wäre dann einfach eine Art Kalkulation, mechanistische Verwendung oder Einsatz des kognitiven Apparats, der kognitiven Fähigkeiten des Menschen. Entscheidung setzt aber immer eine bestimmte Freiheit voraus. Und hinsichtlich solcher Kalkulationen kommt zum anderen die Frage nach der Verantwortung überhaupt nicht länger in Betracht - denn würde man eine Maschine dafür verantwortlich machen, dass ihre Kalkulationen ein bestimmtes Ergebnis liefern?

dungen, und bei Aristoteles um kleine und alltägliche Entscheidungen geht, so dass diese zwei Auffassungen schon deshalb nicht miteinander vergleichbar wären. Dieser Einwand scheint plausibel zu sein, zumal Kierkegaard ausgerechnet Abraham als Beispiel verwendet. Im Hinblick auf Kierkegaard scheint dieser Einwand auch einigermaßen zuzutreffen, für Derrida ist Abraham jedoch nur ein Beispiel, das den „unmöglichen“ Augenblick, durch den jede Entscheidung gehen muss, veranschaulicht; es geht ihm um die Struktur der Entscheidungen, die kleineren Entscheidungen weisen dieselbe Struktur auf wie die sogenannten „existenziellen“. - Darüber hinaus könnte man noch anführen, dass es erstens bei Aristoteles nicht nur um „kleine“ Entscheidungen geht, einige seiner Beispiele behandeln Fragen um Leben und Tod. Zweitens sagt Aristoteles zwar explizit, dass man sich für die Mittel und nicht für die Ziele entscheidet; wenn das Ziel jedoch zu umfangreich gefasst wird, wie z.B. ein glückliches Leben, dann werden die Entscheidungen, die man diesbezüglich treffen muss, „existenziell“. Insofern drittens die Entscheidungen eine Rolle beim Erwerb der Grundhaltung (und somit bei der Aneignung der Ziele) spielen, bestimmen sie das ganze Leben. Viertens sei daran erinnert, dass Entscheidungen nach Aristoteles nicht leicht und schnell getroffen werden können. 
Dazu Derrida: „If my theoretical analysis of the situation shows me what is to be done - do this to cause that, etc.- then there is no moment of decision" (Derrida 2002, 231). Wen man also weiß, was zu tun ist, ist seine Handlung nur eine Anwendung oder ein Einsatz des Wissens. Derrida versucht hier auf explizite und radikale Weise den dem Begriff der Entscheidung inhärenten Bruch zu denken: den „Akt“, der schneidet und abbricht. Dieser Bruch ist in den Begriff der Entscheidung eingeschrieben, was bereits die Etymologie hin zeigt und er tritt auch bei Aristoteles hervor, wenn man ihn genau liest.

Das heißt aber nicht, dass die Entscheidung nichts mit der Überlegung, dem Denken, zu tun hätte. Derrida betont umgekehrt, dass der Entscheidung die Überlegung vorausgehen muss, dass man immer versuchen sollte, so viel wie möglich, ja das Maximum, zu wissen (vgl. Derrida 2000, 40; Derrida 2002). Nur im echten Augenblick der Entscheidung - s'il y en a; wenn es sie gibt ${ }^{14}$-, wo die Entscheidung getroffen wird, muss sie mit jeder Überlegung und mit jeder Art von Gründen, die gleichzeitig an den Tag treten, radikal brechen - der Augenblick der Entscheidung ist immer ein Augenblick der Dringlichkeit und Überstürzung (Derrida 1990, 966). Derrida weist hiermit willentlich auf eine bestimmte Unzugänglichkeit des SichEntscheidens, auf eine aporia hin: Einerseits sollte eine Überlegung und ein Wissen jeder Entscheidung vorausgehen, andererseits muss diese, um Entscheidung zu sein, im selben Augenblick mit jeder Art von Wissen brechen. Oder ein wenig anders und im Hinblick auf die genuin ethischen Entscheidungen ausgedrückt: um ethisch und verantwortlich zu handeln, darf man nicht einfach und ohne weiteres den ethischen Vorschriften folgen - das wäre eben unverantwortlich, denn es würde wieder heißen, dass man gemäß einem Programm und wie eine Maschine handelt. ${ }^{15}$ Die allgemeinen ethischen Vorschriften können darüber hinaus dem singulären Anderen gegenüber nicht gerecht werden, da er in ihnen nicht aufgeht. Zugleich muss man aber die allgemeine Moral in Betracht ziehen und sich in seinen Entscheidungen an ihr orientieren. Andernfalls wäre der singuläre Andere jeglicher Willkür ausgesetzt. Abrahams Entscheidung, seinen Sohn zum Opfer zu bringen, musste durch diese Spannung, zwischen dem Ruf des singulä-

${ }^{14}$ Derrida fügt fast immer, wenn er von Entscheidung oder Verantwortung spricht, dieses s'il y en a hinzu - er möchte damit darauf hinweisen, dass solche Phänomene nicht durch Wissen bestimmt werden können. Er selber äußert sich dazu wie folgt: „I often use the expression s'il y en $a$, when I speak of our relation to choice, decision and responsibility, but this does not mean that these things do not exist or that they are impossible, it means rather that our relation to matters like choice, decision and responsibility is not a theoretical, constative or determinate relation" (Derrida 1996, 87). Vgl. auch (Derrida 2002, 229).

${ }^{15}$ Im Hinblick auf den kategorische Imperativ Kants schreibt Derrida: „If I act in accordance with duty in the Kantian sense, I do not act and furthermore I do not act in accordance with duty" (Derrida 1996, 84). 
ren Anderen und den moralischen Vorschriften, hindurchgehen. Die durch allgemeine Moral geregelte Verantwortung, die Abraham gegenüber Isaak und seiner Frau Sarah hatte, konnte nicht mit dem Ruf des singulären Anderen versöhnt werden. Eben in dieser Spannung zwischen den verschiedenen Pflichten, in diesem Konflikt zwischen dem Allgemeinen und dem Singulären, fangen nach Derrida die ethischen Probleme an.

In Force de loi «le fondement mystique de l'autorité» (1990) erläutert Derrida dies am Beispiel rechtlicher Entscheidungen: einerseits darf der Richter nicht nur und nicht einfach dem Gesetz folgen, denn das wäre nur eine maschinelle Anwendung der Regel und somit auch unverantwortlich und ungerecht. Ungerecht, weil ein partikulärer Fall nicht völlig der allgemeinen Regel zugeordnet werden kann - allgemeine Regeln werden der Singularität der Situation und des Angeklagten nicht gerecht. Auf einer zweiten Ebene kann auch die Gesetzlichkeit des Gesetzes nicht selbst gesetzlich sein, da die Begründung des Grundes unbegründet bleibt - das Gesetz, das beschlossen wurde, konnte nicht im Augenblick seiner Beschließung sich selbst als Maßstab nehmen. Der Akt der Begründung des Gesetzes war weder gesetzlich noch ungesetzlich (das Gesetz war noch nicht da) - und daher le fondement mystique de l'autorité. Kurz gesagt, das Gesetz selber ist arbiträr, grundlos, kontingent und mehr oder minder gewalttätig (vgl. Sokoloff 2005, 343). Im Namen der Gerechtigkeit, die Derrida so streng wie möglich vom Gesetz unterscheidet, müsste man diese Grundlosigkeit und Kontingenz im Auge behalten, so dass eine Verbesserung der Gesetze als offene Möglichkeit bestehen bleibt. Dabei muss sich der Richter, wenn er eine Entscheidung trifft und nicht einfach einem Programm folgen will, auf jene Kontingenz und Grundlosigkeit einlassen, um gegenüber der Singularität des Anderen gerecht zu urteilen. Andererseits muss der Richter als Vertreter der Gesetzmäßigkeit sich natürlich streng an das Gesetz halten. Derrida spricht diesbezüglich oft von der Prüfung des Unentscheidbaren, der sich jede Entscheidung unterziehen muss, um nicht einfach ein berechenbares Vorgehen, eine mechanische Kalkulation zu sein. Das Unentscheidbare ist die kaum wahrnehmbare Suspension der Gründe, des Zwecks, des Rechthandelns, der Überlegung und der Berechnung im Augenblick der Entscheidung. Oder ein wenig anderes ausgedrückt: es ist der Augenblick der Blindheit und des Nicht-Wissens, in dem man nicht weiß, wie man sich entscheiden und handeln soll. Diese Blindheit ist nicht Mangel an Wissen — sie hat nichts damit zu tun, was man im Prinzip wissen könnte -, sondern gehört irreduzibel zur Struktur der Entscheidung (Derrida 2002, 232). Ohne die Erfahrung des Unentscheidbaren könnte man nur von der Anwendung der Regel sprechen. Wie bereits angedeutet, handelt es sich hier nicht um eine neue, wenn auch radikale Interpretation der Entscheidung. Die skizzierten Aporien sind (analytisch) 
im Begriff der Entscheidung selbst angelegt (Derrida 2002, 232) und etymologisch entsprechend impliziert (décision als ,zerreißen` und ,aufschneiden vgl. Derrida 1994, 87).

Zwar ist Derrida kritisiert worden, dass durch die Konzipierung der Entscheidungen über das Unentscheidbare Entscheidungen bereits unmöglich gemacht werden. Man würde bei einer solchen Suspension unendlich verweilen und nie zu einer Entscheidung kommen (vgl. Undusk 1998; Derrida 2002, 230). Dennoch geht dieser Einwand an der Sache vorbei. Bei Derrida kommt es gerade auf die (tatsächliche) Entscheidung an, die folglich eine Suspension und zugleich den Abbruch dieser notwendigen Suspension darstellt: die Entscheidung, und insbesondere die gerechte, angemessene Entscheidung „est toujours requise immédiatement" (Derrida 1990, 966). Paradox bleibt dabei, dass man eine solche Entscheidung nur dann getroffen haben wird, wenn man vom Unentscheidbaren heimgesucht wird. Und diese kafkaeske Heimsuchung wird Derrida zufolge nie völlig vorübergehen und überwunden, sogar wenn die Entscheidung schon getroffen ist das Unentscheidbare wird als Gespenst die Selbstsicherheit jeder Entscheidung untergraben, jedes "gute Gewissen“ in Unruhe versetzen. Das heißt ganz einfach, sich nicht sicher sein zu können, eine gute, verantwortliche, gerechte Entscheidung getroffen zu haben. Man weiß nicht einmal, ob die Entscheidung überhaupt eine war. ${ }^{16}$ In Politiques de l'amitié, aus dessen Vorwort ich oben bereits einige Sätze zitiert habe, spricht Derrida nun anstelle des Unentscheidbaren vom vielleicht:

Sans louverture d'un possible absolument indéterminé, sans le suspens radical que marque un peut-être, il n'y aurait jamais ni événement ni décision. Certes. Mais rien n'arrive et rien ne se décide jamais qu'à lever le peut-être en en gardant la possibilité «vivante», en mémoire vive. (Derrida 1994, 86)

Hier kann man noch deutlicher sehen, dass Derrida mit dem Unentscheidbaren nicht beabsichtigt, Entscheidungen unendlich zu verschieben. Umgekehrt: mit der Bedingung der Möglichkeit der Entscheidung, mit dem Vielleicht, dem Unentscheidbaren, muss - wie paradox es auch klingen mag - gebrochen werden, um Entscheidung überhaupt treffen oder fällen zu können, d .h. die Bedingung der Möglichkeit der Entscheidung ist zugleich die Bedingung der Unmöglichkeit der Entscheidung. Aber selbst

${ }^{16}$ In Derridas eigenen Wörtern: „L'indécidable reste pris, logé, comme un fantôme au moins, mais un fantôme essentiel, dans toute décision, dans tout événement de décision. Sa fantomaticité déconstruit de l'intérieur toute assurance de présence, toute certitude ou toute prétendue critériologie nous assurant de la justice d'une décision, en vérité de l'événement même d'une décision“ (Derrida 1990, 964). 
dann, wenn dieses Vielleicht suspendiert wurde, bleibt es als eine „lebendige Möglichkeit" und raubt jeder Entscheidung ihre Selbst-Sicherheit.

Man könnte an dieser Stelle die Frage stellen, warum Derrida solche Phänomene wie Entscheidung oder Verantwortlichkeit zu Aporien drängen muss. Welche Beweggründe gibt es dafür? Einerseits handelt es sich, wie Derrida wiederholt betont, um eine Bewegung innerhalb der wesentlichen und wichtigen Begriffe und Texte selbst. Von daher sei man - als Philosophzur wiederholten und genauen Lektüre verpflichtet. Die genannten Aporien treten im Text jedoch nie eigens hervor, sie,wesen' nicht, an' und sind auch nicht vollständig in ein phänomenales Licht zu bringen. Phänomene wie Entscheidung oder Verantwortung entziehen sich daher dem theoretischen Wissen. Aporien markieren die Grenze des begrifflichen Denkens über solche Phänomene. Andererseits sind Aporien die Augenblicke der Krisis und Angst - durch solche aporetische Auffassungen, wie schon betont, werden alle guten Gewissen erschüttert (vgl. Sokoloff 2005, 344). Man kann nicht mehr wissen, ob es Entscheidung gab und ob es gut und richtig war (vgl. Derrida 2002, 232; Derrida 1996, 86; Derrida 2003, 43). Eine solche Unruhe ist nach Derrida die treibende Kraft innerhalb der Ethik und des Politischen, der schrittweisen Verbesserung der Politik.

Das Unentscheidbare als ein Moment des Nicht-Wissens, der strukturellen Blindheit, bringt sicherlich ein bestimmtes Risiko in die Entscheidung (und Politik) ein. Es gibt sogar die Meinung, dass Derrida Schmitts Dezisionismus vertritt (vgl. Lilla 2001), wonach eine Entscheidung jenseits von allen rechtlichen Normen und Rationalität, der Vernunft, getroffen werden muss. Doch Derrida distanziert sich deutlich von Schmitts Dezisionismus als der Theorie des Feindes, die immer noch ein klassisches Subjekt voraussetzt (vgl. Derrida 1994, 87). Andererseits muss, wie wir gesehen haben, eine (richterliche) Entscheidung bei Derrida auch eine Beziehung - selbst wenn sie aporetisch ist - zum Gesetz, zur Überlegung und zu den allgemeinen ethischen Vorschriften haben (vgl. Sokoloff 2005, 344). Der Richter darf eben nur nicht einfach dem Gesetz folgen, weil das die Verantwortlichkeit vor dem singulären Anderen herabsetzt bzw. beseitigen würde. Nach Nick Mansfield unterscheidet Derrida von Schmitt gerade diese durcheinanderbringende Rolle des Anderen und zugleich die Beängstigung und Unruhe (in-competence), die jede "derridaische“ „Entscheidung" mit sich bringt (Mansfield 2006, 482). ${ }^{17}$ Das heißt jedoch nicht, dass das Risiko damit vollständig beseitigt wäre. Derrida schreibt: „Sans la possibilité du mal radical, du parjure et du crime absolu, aucune responsabilité, aucune liberté, aucune décision“ (Derrida 1994, 247).

${ }^{17}$ Für eine ausführliche Behandlung der Beziehung zwischen Derrida und Schmitt vgl. (Ungureanu 2008, 293-325). 


\section{2 „Subjekt“ und der Andere}

Wenden wir uns nun der Frage zu, inwiefern die Entscheidung nach Derrida vom ,Subjekt' getroffen wird. Letzteres wird, wie bereits angedeutet, als ein bestimmter und mehr oder minder (selbst)sichere Grund (der Entscheidung) verstanden. Unter den derridaischen Prämissen kann das,Subjekt' nie etwas entscheiden. Warum nicht? In der philosophiegeschichtlichen Tradition werden dem ,Subjekt' bestimmte und mehr oder weniger beständige Eigenschaften zugewiesen. Es weist eine Grundhaltung auf und verfügt über darauf basierende Möglichkeiten, so dass die „Entscheidung“ eines Subjekts immer gemäß diesen Eigenschaften getroffen worden wäre - kurz, sie wäre eine Entfaltung der Möglichkeiten eines ,Subjektes', eines Charakters. Letzteres wäre aber wiederum nur eine Befolgung eines Programmes. Entscheidung kann nicht einfach Ausdruck meiner Macht, meiner Möglichkeiten sein (Derrida 2003, 43), vielmehr müsste Ent-scheidung (décision) als Zerreißen den Rahmen des Möglichen sprengen (Derrida 2003, 44). Könnte man in Erfahrung bringen, wer und worüber er / sie entscheidet, also wenn es „Subjekt" und „Objekt" gäbe, so würde man Entscheidung auf eine application of a law reduzieren (Derrida 1996, 84). Der ethische Aspekt ist noch einmal unübersehbar: ein ,Subjekt' als bestimmte Beständigkeit und Identität kann mit seinen „Entscheidungen“ dem Anderen nicht gerecht werden, es kann ihm nie so antworten, dass es sich dabei gleichzeitig vollständig transformieren würde (vgl. Sokoloff 2005, 345). Man könnte Derridas Einsichten mit denjenigen Aristoteles vergleichen, wonach die Richtigkeit der Entscheidung von den Tugenden abhängt, die als feste Grundhaltungen der Mitte (zwischen den extremen Handlungsweisen) eine Garantie für die richtige Entscheidung sind - nach Derrida wäre eine solche Gewährleistung gerade das Vermeiden der "richtigen“ Entscheidung. Um auf den Ausgangspunkt dieses Aufsatzes zurückzukommen, scheint die Auffassung von Aristoteles in einem gewissen Sinne tatsächlich auf die Vermeidung bzw. ein Ausweichen vor der Entscheidung hinauszulaufen.

Das ,Subjekt' entscheidet folglich nicht, es verhält sich gerade umgekehrt: das ,Subjekt' wird durch jede Entscheidung wieder neu erfunden, d. h. überrascht, erschüttert und in einem bestimmten Sinne neu gestaltet (vgl. Derrida 1996, 84; Derrida 1994, 87). Doch wer kann dann überhaupt entscheiden und / oder woher kommt die Entscheidung? Hier knüpft Derrida an Levinas an setzt sich aber auch ein stückweit von ihm ab. ${ }^{18}$ Die Entschei-

${ }^{18}$ Zur Beziehung zwischen Derrida und Levinas vgl. (Derrida 1967), (Derrida 1995), (Derrida 1997), (Critchley 1992). Vgl. auch Hans Kimmerle (2005, 57): „Der Unterschied zu Levinas besteht im Grunde darin, dass Derrida den vertikal begründeten Appell des Anderen bei Levinas, der nicht in eine zwischenmenschliche Beziehung zu übersetzen ist, in eine horizontale Denkrichtung umzubiegen sucht." 
dung rühre vom anderen her, und zwar im Entscheiden selbst: Der Augenblick, wo ich mich um des anderen willen entscheide, ist zugleich der Augenblick, wo ich ihn umso besser verfehlt haben könnte. Die Entscheidung ist somit alles andere als ein aktiver und souveräner Akt, wie sie immer vorgestellt wurde, sondern etwas "Passives“ und teilweise sogar Unbewusstes. Sie bleibt aber dennoch verantwortlich. Diese „Passivität" folgt unweigerlich aus dem bereits Gesagten: denn wenn jemand beim Treffen der Entscheidung aktiv wäre, d. h. die Aufsicht über den Entscheidungsakt behielte, dann würde er sich die Entscheidung zu eigen machen und sagen: „es ist meine Entscheidung"; und wenn sie seine ist, dann heißt das, dass sie seine eigene Möglichkeit entfaltet (Derrida 2000, 39). Die Entscheidung, wie Derrida immer wieder betont, dürfe jedoch nicht einfach einem Programm folgen. In Politique de l'amitié schreibt er:

Elle [décision passive] signifie donc l’autre en moi qui décide et déchire. La décision passive, condition de l'événement, c'est toujours en moi, structurellement, une autre décision, une décision déchirante comme décision de l'autre. ... Une décision est inconsciente en som$m e$, si insensé que cela paraisse, elle comporte l'inconscient et reste pourtant responsable. (Derrida 1994, 87-88)

Die Entscheidung kann nicht meine sein, sie muss mit meiner Macht, meinem Vermögen brechen. Aber eben das ist unmöglich: wenn ich mit meinen Möglichkeiten brechen würde (vorausgesetzt, dies ist überhaupt möglich), so läge dieser Bruch immer noch im Bereich meiner Möglichkeiten. Diese Aporie und Unzugänglichkeit der begrifflichen Auffassung der Entscheidung haben wir mehrmals wahrgenommen. Nun scheint es, dass Derrida einen Weg durch diese begriffliche Aporie eröffnen möchte, nämlich eben dadurch, dass er eine Entscheidung als Entscheidung des Anderen thematisiert. Um möglich zu sein, muss die Entscheidung vom Anderen in mir herkommen, nur der / die / das Andere kann den Entscheidenden radikal erschüttern, dessen Macht und Möglichkeiten brechen. Wie hängt das aber mit der Aporie des Unentscheidbaren zusammen? Das Unentscheidbare war ein Augenblick des Nicht-Wissens, der strukturellen Blindheit, und der Andere bricht nun gerade in der Erfahrung dieses aporetischen Augenblicks, in dem man nicht weiß, was zu tun und was zu meiden ist, in den Entscheidenden ein, bricht mit seinem Charakter und seiner Macht, indes er eine Entscheidung fällt. Diese Entscheidung als Entscheidung des Anderen im Entscheidenden ist Derrida zufolge, wie man im Zitat lesen kann, teilweise unbewusst. Das heißt, dass die Entscheidung nicht autonom, sondern heteronom verläuft - für ein traditionelles Verständnis der Entscheidung eine ungeheure Schlussfolgerung. Nehmen wir noch einmal Abraham als Beispiel: Man kann sagen, dass der völlig Andere, Gott, der in Abraham ist, 
teilweise unbewusst ist, in jenem Moment, in dem Abraham das absolute Nicht-Wissen im Hinblick auf seine Entscheidung ertragen muss, das Unentscheidbare suspendiert und für Abraham entscheidet, dass er seinen eigenen Sohn zum Opfer bringen soll. Für seine Tat bleibt Abraham „selber“" verantwortlich, wie paradox das auch klingen mag: „responsable de l'autre devant lautre" (Derrida 1994, 87-88). Abraham ist vom Anderen gerufen und aufgefordert, etwas Unmögliches zu tun. Indem er es tut, ist es auch nicht länger unmöglich. In Abrahams Fall wäre es einfach nur ein Mord. Und für diesen wäre Abraham verantwortlich. Diese Verantwortung ist jedoch genauso wenig selbst übernommen wie die Entscheidung — sie ist Abraham vom Anderen zugeschrieben worden. Nun nach Derrida ist natürlich jeder Mensch (und auch das Tier z. B., also nicht nur Gott) dieser völlig Andere: tout autre est tout autre ${ }^{19}$ - jeder Andere ist ganz Anderer (Derrida 1995, 78).

Die Thematik des Anderen ist vielfältig bei Derrida und, wie gesagt, auch Levinas hat ihn in dieser Hinsicht erheblich beeinflusst. Der Mensch ist nach Derrida von Beginn an von einer ursprünglichen Sozialität oder Gesellschaftlichkeit gefangen oder, mit seinen eigenen Wörtern ausgedrückt: „before even having taken responsibility ... we are already caught up in a kind of asymmetrical and heteronomical curvature of the social space, ... in the relation to the Other prior to any organized socius, to any determined "government" ..." (Derrida 1988, 633 f.). Bevor wir irgendeine sogenannte autonome Beziehung zu den Anderen haben, Verantwortung übernehmen können, bevor diese Beziehung durch Gesetz und Gesellschaft vermittelt worden ist, stehen wir schon vom Anfang an vor den Anderen, vor dem Ruf der Anderen, müssen den Anderen antworten, sind durch die Spuren der Anderen konstituiert. Unsere „Autonomie“ ist eine Heteronomie. Gerade in diesem Kontext soll die passive Entscheidung als Entscheidung des Anderen verstanden werden.

\section{Schlusswort}

Derrida hat auf rigorose und radikale Weise den Bruch zu bestimmen versucht, von dem die traditionelle Auffassung der Entscheidung immer schon affiziert ist. Bereits Aristoteles hat Entscheidung derart verstanden, dass ein Mensch dadurch zum Handlungsursprung, zum Anfang seiner Handlungen wird - von daher fehlt zumindest die Silhouette des radikalen Bruches und des Anfangs nicht bei ihm. Jedoch hat Aristoteles die Entscheidung auf die Überlegung reduziert und somit den Bruch, das Unentscheidbare, quasi überdeckt. Man muss es nicht sehen. Dasselbe gilt für die Freiheit -

\footnotetext{
${ }^{19} \mathrm{Zu}$ diesem Ausdruck und seiner Beziehung zu Levinas vgl. (Pfeuffer 2008, 216-220).
} 
der Begriff der Entscheidung setzt diese voraus, schon bei Aristoteles. Man könnte einwenden, wie frei eine Entscheidung ist, wenn sie einerseits aus der Grundhaltung und andererseits unmittelbar aus der Überlegung folgt. Für Derrida wäre dies die Befolgung eines Programmes, eine maschinelle Handlung, die von der Verantwortung entlastet. Gerade in Hinblick auf diesen Bruch mit irgendeinem Programm (Ethik, Gesetz, Gründe) ist die Entscheidung eine Häresie, hairesis (,Wahl') oder prohairesis. Derrida radikalisiert die Freiheit, die freilich nicht länger meine Freiheit ist, sondern vielmehr Freiheit von mir, eine Freiheit, die vom Anderen in mir herkommt und mit meinen Möglichkeiten und Kalkulationen bricht. Die Entscheidung ist daher etwas Passives.

Was die Entscheidung so schwierig bei Derrida macht, ist, dass sie gleichzeitig einen wesentlichen Bezug auf die Überlegung, Vernunft, ethische Vorschriften und Gesetz behalten muss. Auch dieses, d. h. die ernste Überlegung, hat der Begriff der Entscheidung immer schon impliziert. Aristoteles ist hierfür ein gutes Beispiel. Der Entscheidende muss überlegen, so muss der Richter z. B. dem Gesetz folgen. Wenn er dies jedoch in automatischer Weise und ohne die kaum wahrnehmbare Suspension durch das Unentscheidbare tun würde, dann würde er letztlich auch nicht über den Anderen urteilen. Er würde die Andersheit des anderen nicht berücksichtigen und damit kein gerechtes Urteil fällen. Der Richter selbst würde den „mystischen“ Moment der Errichtung des Gesetzes verkennen und die Möglichkeit der Veränderung des Gesetzes ausschließen. Eben weil die Gründung des Gesetzes durch das Unentscheidbare hindurchgehen, es aber an einem bestimmten Punkt seinerseits verkennen muss, kann es sowohl Gesetze als auch die Möglichkeit ihrer Veränderung geben. Von daher hat Derridas Auffassung der Entscheidung einen unbestreitbaren ethischen Aspekt. Wenn jedoch das Unentscheidbare ein Augenblick des strukturellen Nicht-Wissens ist, in dem der Andere in mir unbewusst in mich ,eingreift', ist das Risiko einer absoluten Verfehlung nicht zu leugnen oder zu verheimlichen. Das wäre eben der Preis jeder „Verbesserung“.

\section{Danksagung}

Für die gründliche Lektüre der früheren Fassungen dieses Aufsatzes möchte ich Silvio Pfeuffer und Marta Faustino danken. 


\section{Literatur}

Aristoteles (1995). Erste Analytik, in Aristoteles, Philosophische Schriften I, Felix Meiner Verlag, Hamburg.

Aristoteles (2006). Nikomachische Ethik, Reclam, Stuttgart.

Aristotle (1935). Eudemian Ethics, in Aristotle, The Athenian Constitution. The Eudemian Ethics. On Virtues and Vices, Harvard University Press, Cambridge.

Aristotle (2002). Nicomachean Ethics, Oxford University Press, Oxford.

Broadie, S. (2002). Philosophical introduction, in Aristotle, Nicomachean Ethics, Oxford University Press, Oxford, S. 9-91.

Cosmus, O. (2001). Anonyme Phänomenologie. Die Einheit von Heideggers Denkweg, Königshausen \& Neumann, Würzburg.

Critchley, S. (1992). The Ethics of Deconstruction: Derrida and Levinas, Blackwell, Oxford.

Derrida, J. (1967). Violence et métaphysique: Essai sur la pensée d'Emmanuel Levinas, in J. Derrida L'écriture et la différence, Seuil, Paris, S. 117-229.

Derrida, J. (1988). The politics of friendship, The Journal of Philosophy 85: 632-644.

Derrida, J. (1990). Force de loi: «le fondement mystique de l'autorité », Cardozo Law Review 11: 919-1045.

Derrida, J. (1994). Politiques de l'amitié, Editions Galilée, Paris.

Derrida, J. (1995). The Gift of Death, University of Chicago Press, Chicago.

Derrida, J. (1996). Remarks on deconstruction and pragmatism, in C. Mouffe (hrsg.), Deconstruction and Pragmatism, Routledge, London, S. 77-88.

Derrida, J. (1997). Adieu à Emmanuel Lévinas, Galilée, Paris.

Derrida, J. (200o). As If I were Dead - Als ob ich tot wäre, Turia und Kant Verlag, Wien.

Derrida, J. (2002). Nietzsche and the machine, in E. Rottenberg (hrsg.), Negotiations: Interventions and Interviews, 1971-2002, Stanford University Press, Stanford, S. 215-256.

Derrida, J. (2003). Eine gewisse unmögliche Möglichkeit, vom Ereignis zu sprechen, Merve Verlag, Berlin.

Heidegger, M. (1989). Beiträge zur Philosophie (vom Ereignis), in F.-W. v. Herrmann (hrsg.), Gesamtausgabe 65, Vittorio Klostermann, Frankfurt am Main. 
Kierkegaard, S. (1992). Furcht und Zittern, Europäische Verlagsanstalt, Hamburg.

Kimmerle, H. (2005). Jacques Derrida interkulturell gelesen, Traugott Bautz, Nordhausen.

Kuhn, H. (1960). Der Begriff der Prohairesis in der Nikomachischen Ethik, in D. Henrich, W. Schulz and K.-H. Volkmann-Schluck (hrsg.), Die Gegenwart der Griechen im neueren Denken: Festschrift für Hans-Georg Gadamer zum 6o. Geburtstag, J.C.B. Mohr, Tübingen, S. 123-140.

Lilla, M. (2001). The Reckless Mind: Intellectuals in Politics, New York Review of Books, New York.

Mansfield, N. (2006). Refusing defeatism: Derrida, decision and absolute risk, Social Semiotics 16: 473-483.

Pfeuffer, S. (2008). Die Entgrenzung der Verantwortung: NietzscheDostojewskij-Levinas, Walter de Gruyter, Berlin.

Rapp, C. (1995). Freiwilligkeit, Entscheidung, Verantwortlichkeit, in O. Höffe (hrsg.), Aristoteles: Die Nikomachische Ethik, Akademie Verlag, Berlin, S. 127-133. Klassiker Auslegen, Bd. 2.

Rawls, J. (1993). Political Liberalism, Columbia University Press, New York.

Robinson, R. (1995). Aristotle on Akrasia, in O. Höffe (hrsg.), Aristoteles: Die Nikomachische Ethik, Akademie Verlag, Berlin, S. 187-206. Klassiker Auslegen, Bd. 2.

Ross, D. (1985). Aristotle, Methuen \& Co, New York.

Schmidt, E. (2006). Anmerkungen, in Aristoteles, Nikomachische Ethik, Reclam, Stuttgart, S. 303-353.

Sokoloff, W. (2005). Between justice and legality: Derrida on decision, Political Research Quarterly 58: 341-352.

Undusk, J. (1998). Maagiline müstiline keel, Virgela, Tallinn.

Ungureanu, C. (2008). Derrida on free decision: Between Habermas' discursivism and Schmitt's decisionism, The Journal of Political Philosophy 16: $293-325$. 


\section{Decision as Heresy}

It might be said that Aristotle institutes something like a traditional and dominating concept of decision in the philosophical tradition of the West. The aim of this article is to outline that concept of Aristotle, touching on some of its most important problems, and contrast it with what I would call Jacques Derrida's heretical understanding of decision. It will be shown that this heretical "conceptualization" by Derrida does not simply and merely break with, or depart and differ from, the traditional Aristotelian concept but rather also justifiably sharpens some of its most central features in such a way that from that radicalized point of view the treatment of Aristotle comes to appear as the very annulment of decision. It is moreover implied how Derrida's heretical understanding of decision entails a renewal of ethics and politics.

Keywords: ethics, decision, undecidability, freedom, responsibility, subject, deliberation, law, aporia 